TRANSACTIONS OF THE

AMERICAN MATHEMATICAL SOCIETY

Volume 263, Number 2, February 1981

\title{
CAPACITY DENSITIES AND ANGULAR LIMITS OF QUASIREGULAR MAPPINGS
}

BY

\author{
MATTI VUORINEN
}

\begin{abstract}
It is shown that if a bounded quasiregular mapping of the unit ball $B^{n} \subset R^{n}, n>2$, has a limit at $b \in \partial B^{n}$ through a set $E \subset B^{n}$ with $b \in \bar{E}$, then it has an angular limit at $b$ provided that $E$ is contained in an open cone $C \subset B^{n}$ with vertex $b$ and that $E$ is thick enough at $b$. The thickness condition is expressed in terms of the $n$-capacity density.
\end{abstract}

1. Introduction. Quasiregular (qr) mappings in $R^{n}$ can be regarded as a generalization of the notion of an analytic function in the complex plane (cf. [7], [8], [15]). In this paper we shall prove some results on boundary behavior of qr mappings, which, to a certain extent, are analogous with known properties of analytic functions and in the particular case of injective qr mappings follow from the results proved in [17]. We remark that the class of injective qr mappings is the same as the class of sense-preserving quasiconformal (qc) mappings [14].

A bounded analytic function of the unit disc having a limit $\alpha$ through a curve terminating at a boundary point $b$ has angular limit $\alpha$ at $b$ according to a classical theorem of Lindelöf. Recently Rickman [13] constructed an example of a bounded qr mapping of the $n$-dimensional unit ball $B^{n}, n \geqslant 3$, which has a limit through a curve terminating at a boundary point but has no angular limit at the point in question. Therefore a direct analogue of Lindelöf's theorem fails to hold for bounded quasiregular mappings of $B^{n}$ when $n \geqslant 3$.

The following positive result will be proved in $\$ 3$ of this paper. Let $f: B^{n} \rightarrow B^{n}$, $n \geqslant 2$, be qr, $b \in \partial B^{n}$, and let $C \subset B^{n}$ be an open cone with vertex $b$. Let $E \subset C$ and cap dens $(E, b)>0$. If $\lim _{x \rightarrow b, x \in E} f(x)=0$, the mapping $f$ has angular limit 0 at $b$. The condition cap dens $(E, b)>0$ is best possible in the sense of Remark 3.2(2). Here cap dens refers to the lower $n$-capacity density [9], [5]. The condition is satisfied e.g. if $\overline{E \text { is a }}$ curve. Thus we have established a Lindelöf-type theorem for curves approaching a boundary point within a cone. In $\$ 4$ we shall weaken the assumption that the mapping be bounded.

A crucial tool for this paper is a local version of a two-constants theorem for $\mathrm{qr}$ mappings, which Rickman [12, 4.22] has derived from some estimates for the solutions of elliptic partial differential equations due to Mazja. An appropriate iterated version of the two-constants theorem is given in \$2. It essentially accomplishes the iteration ideas of Rickman [12, 4.24] in the quasihyperbolic geometry of

Received by the editors February 15, 1980; presented to the Society, January 5, 1980.

1980 Mathematics Subject Classification. Primary 30C60; Secondary 30D40.

Key words and phrases. Quasiconformal and quasiregular mappings, boundary behavior, angular limits. 
an arbitrary domain $G \neq R^{n}$. The use of the quasihyperbolic geometry (GehringPalka [2]) makes it possible to pass from local to global estimates. The main results of the paper, proved in $\$ \S 3$ and 4, were announced in a lecture [19] given during the Complex Analysis Semester at the Banach Center (Warsaw, Poland) in May 1979. A summary of the results in [17], [18] can also be found in [19].

2. Preliminary results. We first introduce some terminology and notation.

2.1. For $x \in R^{n}(n \geqslant 2)$ and $r>0$ let $B^{n}(x, r)=\left\{y \in R^{n}:|y-x|<r\right\}$, $S^{n-1}(x, r)=\partial B^{n}(x, r), B^{n}(r)=B^{n}(0, r), S^{n-1}(r)=\partial B^{n}(r), B^{n}=B^{n}(1)$, and $S^{n-1}$ $=\partial B^{n}$. If $r>s>0$, then we write $R(x, r, s)=B^{n}(x, r) \backslash \bar{B}^{n}(x, s)$ and $R(r, s)=$ $R(0, r, s)$. The standard coordinate unit vectors are $e_{1}, \ldots, e_{n}$.

2.2. Quasiregular mappings. Let $G \subset R^{n}$ be a domain and let $f: G \rightarrow R^{n}$ be a continuous mapping in the local Sobolev space $W_{n, \text { loc }}^{1}$. Then $f$ is said to be $K$-quasiregular $(K$-qr) if there exists a constant $K \geqslant 1$ such that the following two inequalities,

$$
\sup _{|h|=1}\left|f^{\prime}(x) h\right|^{n} \leqslant K J_{f}(x), \quad J_{f}(x) \leqslant K \inf _{|h|=1}\left|f^{\prime}(x) h\right|^{n},
$$

hold a.e. in $G$. Here $J_{f}$ is the Jacobian determinant of $f$ and $f^{\prime}(x): R^{n} \rightarrow R^{n}$ is the linear mapping $f^{\prime}(x) e_{i}=\partial f(x) / \partial x_{i}, i=1, \ldots, n$. Note that both $f^{\prime}(x)$ and $J_{f}(x)$ exist a.e. in $G$ because $f \in W_{n \text {,loc }}^{1}$. The smallest $K$ for which the inequalities are true is denoted by $K(f)$. The mapping $f$ is $K$-quasiconformal $(K$-qc) if it is a $K$-qr homeomorphism. The mapping $f$ is qr (qc) if it is $K$-qr $(K-\mathrm{qc})$ for some $K \in[1, \infty)$. For the basic properties of qc and qr mappings the reader is referred to [7], [8], [15].

2.3. Modulus of a curve family. Let $\Gamma$ be a family of curves in $R^{n}$. The modulus of $\Gamma$ is defined by

$$
M(\Gamma)=\inf _{\rho} \int_{R^{n}} \rho^{n} d m
$$

where $m$ is the $n$-dimensional Lebesgue measure and the infimum is taken over all nonnegative Borel-functions $\rho: R^{n} \rightarrow R^{1} \cup\{\infty\}$ with $\int_{\gamma} \rho d s \geqslant 1$ for all $\gamma \in \Gamma$. Some properties of the modulus will be needed (cf. [14, Chapter 1]). If $E, F$, and $G$ are subsets of $R^{n}$, then we write $\Delta(E, F ; G)=\{\gamma:[0, c) \rightarrow G: \gamma$ continuous, nonconstant, $\gamma(0) \in E, \gamma(t) \rightarrow F$ as $t \rightarrow c\}$. The following lower bound for the modulus will be useful. Let $b>a>0$ and $F_{1}, F_{2} \subset \bar{B}^{n}(a), F_{3} \subset R^{n} \backslash B^{n}(b)$ and write $\Gamma_{i j}=\Delta\left(F_{i}, F_{j} ; R^{n}\right), 1 \leqslant i, j \leqslant 3$. Then (cf. [10, 3.3], [8, 3.11], [17, 1.11])

$$
M\left(\Gamma_{12}\right) \geqslant 3^{-n} \min \left\{M\left(\Gamma_{13}\right), M\left(\Gamma_{23}\right), c_{n} \log (b / a)\right\}
$$

and here $c_{n}$ is a positive constant depending only on $n[14,10.10]$.

2.5. Condenser and its capacity. A pair $(A, C)$ is said to be a condenser if $A \subset R^{n}$ is open and $C \subset A$ is compact. The capacity of $(A, C)$ is defined by

$$
\operatorname{cap}(A, C)=\inf _{u} \int_{R^{n}}|\nabla u|^{n} d m
$$

where $u$ runs through all $C_{0}^{\infty}(A)$ functions with $u(x) \geqslant 1$ for $x \in C$. Alternatively, it can be defined by

$$
\operatorname{cap}(A, C)=M(\Delta(C, \partial A ; A))=M\left(\Delta\left(C, \partial A ; R^{n}\right)\right) .
$$


In the case of bounded $A$ this equality was proved in [20] and a simple argument shows that it is true for nonbounded $A$ as well. Let $f: G \rightarrow R^{n}$ be a nonconstant qr mapping and let $E=(A, C)$ be a condenser with $A \subset G$. Then $f E=(f A, f C)$ is a condenser because $f$ is open and by $[7,7.1]$ we have

$$
\text { cap } f E \leqslant K(f) \text { cap } E \text {. }
$$

2.8. Quasihyperbolic metric. For $A, B \subset R^{n}$ let $d(A)$ and $d(A, B)$ denote the diameter of $A$ and the distance between $A$ and $B$, respectively, in the euclidean geometry. From now on we shall use $G$ to denote a proper subdomain of $R^{n}$. For $x \in G$ let $d(x)=d(x, \partial G) \in(0, \infty)$. Following Gehring and Palka [2, §2] we consider for $a, b \in G$ the number

$$
k_{G}(a, b)=\inf _{\gamma} \int_{\gamma} d(x)^{-1} d s
$$

where $\gamma$ runs through all rectifiable curves with $a, b \in \gamma$. It is clear that $k_{G}$ is a metric in $G$ (note: $G \neq R^{n}$ ), called the quasihyperbolic metric in $G$. If $A \subset G$ then we write $k_{G}(A)=\sup \left\{k_{G}(x, y): x, y \in A\right\}$. From [2, 2.1] it follows that

$$
\begin{gathered}
k_{G}(x, y) \geqslant|\log (d(y) / d(x))|, \\
k_{G}(x, y) \geqslant \log (1+|x-y| / d(x)),
\end{gathered}
$$

for all $x, y \in G$.

For the proof of the main lemma, Lemma 2.22, we shall need several auxiliary results, some of which seem to be of independent interest (especially Lemmas 2.14 and 2.17).

2.11. Lemma. Let $x \in G$ and $y \in B^{n}(x, d(x))$. Then

$$
k_{G}(x, y) \leqslant \log \left(1+\frac{|x-y|}{d(x)-|x-y|}\right) .
$$

Proof. Fix $z \in \partial G$ such that $|x-z|=d(x)$. Denote by $[u, v]$ the closed segment with endpoints $u, v \in R^{n}$. Choose $\bar{y}$ such that $[x, \bar{y}] \subset[x, z]$ and $|x-y|$ $=|x-\bar{y}|$. We estimate

$$
\begin{aligned}
k_{G}(x, y) & \leqslant \int_{[x, y]} d(u)^{-1} d s \leqslant \int_{[x, \bar{y}]} d(u)^{-1} d s \\
& =\log \frac{d(x)}{d(x)-|x-\bar{y}|}=\log \left(1+\frac{|x-y|}{d(x)-|x-y|}\right) .
\end{aligned}
$$

2.12. REMARKS. (1) Given $a, b \in G$, there exists a geodesic curve of the quasihyperbolic metric joining $a$ to $b[1$, Lemma 1]. This means that there exists a rectifiable curve $\gamma$ with $a, b \in \gamma$ and

$$
k_{G}(a, b)=\int_{\gamma} d(x)^{-1} d s .
$$

By considering the case $G=R^{n} \backslash\{0\}, a=e_{1}, b=-e_{1}$, we see that the geodesic curves need not be unique.

(2) If $G^{\prime} \subset G$ is a domain, then clearly $k_{G}(x, y) \leqslant k_{G^{\prime}}(x, y)$ for $x, y \in G^{\prime}$. 
(3) Note that $k_{R_{+}^{n}}$ is the usual hyperbolic metric of the upper half space $R_{+}^{n}=\left\{x \in R^{n}: x_{n}>0\right\}$ defined by the element of length $d s=|d x| / x_{n}$.

(4) Let $\gamma \in G$ be a curve. Then $k_{G}(\gamma) \leqslant s(\gamma) / d(\gamma, \partial G)$ where $s(\gamma)$ is the (euclidean) length of $\gamma$.

(5) Let $F \subset G$ be connected with $\bar{F} \subset G$. For $x, y \in F$ let

$$
s_{x y}=\inf \{s(\gamma): \gamma \subset F \text { is a curve with } x, y \in \gamma\}
$$

and $s(F)=\sup \left\{s_{x y}: x, y \in F\right\}$. Then $k_{G}(F) \leqslant s(F) / d(F, \partial G)$.

Let $a, b \in G$, let $\lambda \in(0,1)$, and let $\left\{B^{n}\left(x_{j}, r_{j}\right): j=1, \ldots, p\right\}$ be a finite family of balls in $G$. The family is said to be $(a, b, \lambda)$-admissible if the following conditions are satisfied.

$$
\begin{aligned}
& \text { (1) } a \in \bar{B}^{n}\left(x_{1}, \lambda r_{1}\right), \quad b \in \bar{B}^{n}\left(x_{p}, \lambda r_{p}\right) \\
& \text { (2) } \bar{B}^{n}\left(x_{j}, \lambda r_{j}\right) \cap \bar{B}^{n}\left(x_{j+1}, \lambda r_{j+1}\right) \neq \varnothing \text { for } j=1, \ldots, p-1 .
\end{aligned}
$$

The importance of the quasihyperbolic metric for what follows is largely due to the following lemma, called here the chaining lemma, which shows that the smallest possible number of balls in an $(a, b, \lambda)$-admissible family is proportional to $c_{\lambda} k_{G}(a, b)$ where $c_{\lambda}>0$ depends only on $\lambda$.

2.14. LEMMA. Let $a, b \in G$ and $\lambda \in(0,1)$.

(1) If $\left\{B^{n}\left(x_{j}, r_{j}\right): j=1, \ldots, p\right\}$ is an $(a, b, \lambda)$-admissible family then

$$
\begin{array}{r}
p \geqslant k_{G}(a, b) /(2 \log (1 /(1-\lambda))) . \\
\text { If } x_{1}=a \text { and }\left|x_{j+1}-x_{j}\right| \leqslant \lambda r_{j} \text { for } j=1, \ldots, p-1, \text { then } \\
p \geqslant k_{G}(a, b) / \log (1 /(1-\lambda)) .
\end{array}
$$

(2) There exists an $(a, b, \lambda)$-admissible family $\left\{B^{n}\left(x_{j}, r_{j}\right): j=1, \ldots, p\right\}$ with $x_{1}=a, x_{j+1} \in \partial B^{n}\left(x_{j}, \lambda r_{j}\right)$ for $j=1, \ldots, p-1$ and with

$$
p \leqslant 1+k_{G}(a, b) / \log (1+\lambda) \text {. }
$$

Moreover, here $r_{j}=d\left(x_{j}\right)$ and $(1-\lambda) r_{j} \leqslant r_{j+1} \leqslant(1+\lambda) r_{j}$.

Proof. (1) Let $B_{j}=B^{n}\left(x_{j}, r_{j}\right)$ and $G^{\prime}=\cup_{j=1}^{p} B_{j}$. Then $G^{\prime}$ is a domain by (2.13). By Remark 2.12(2) and Lemma 2.11 we get

$$
\begin{aligned}
k_{G}(a, b) & \leqslant k_{G^{\prime}}(a, b) \leqslant \sum_{j=1}^{p} k_{G^{\prime}}\left(\bar{B}^{n}\left(x_{j}, \lambda r_{j}\right)\right) \\
& \leqslant \sum_{j=1}^{p} k_{B_{j}}\left(B^{n}\left(x_{j}, \lambda r_{j}\right)\right) \leqslant 2 p \log \frac{1}{1-\lambda} .
\end{aligned}
$$

Suppose now $x_{1}=a,\left|x_{j+1}-x_{j}\right| \leqslant \lambda r_{j}$. Write $x_{p+1}=b$. We obtain

$$
k_{G}(a, b) \leqslant \sum_{j=1}^{p} k_{G^{\prime}}\left(\left[x_{j}, x_{j+1}\right]\right) \leqslant p \log \frac{1}{1-\lambda} .
$$

(2) Fix a geodesic curve $\gamma$ of the quasihyperbolic metric joining $a$ to $b$ (cf. Remark 2.12(1)). Let $x_{1}=a$ and $x_{j+1} \in \partial B^{n}\left(x_{j}, \lambda d\left(x_{j}\right)\right)$ be such that $x_{j+1}$ belongs to the closure of the $b$-component of $\gamma \backslash \cup{ }_{i=1}^{j} \bar{B}^{n}\left(x_{i}, \lambda d\left(x_{i}\right)\right)$. Define 


$$
p-1=\max \left\{j: b \notin \bigcup_{i=1}^{j} \bar{B}^{n}\left(x_{i}, \lambda d\left(x_{i}\right)\right)\right\}<\infty .
$$

Then

$$
k_{G}(a, b)=\int_{\gamma} d(u)^{-1} d s \geqslant \sum_{j=1}^{p-1} k_{G}\left(x_{j}, x_{j+1}\right) \geqslant(p-1) \log (1+\lambda)
$$

and here in the last step we have used (2.10). These estimates yield the desired upper bound for $p$. The estimates for the numbers $r_{j}$ follow from the fact that if $x \in G$ then $(1-\lambda) d(x) \leqslant d(y) \leqslant(1+\lambda) d(x)$ is valid for $y \in \bar{B}^{n}(x, \lambda d(x))$.

For $x \in G, M>0$ we write $D^{k}(x, M)=\left\{z \in G: k_{G}(x, z)<M\right\}$. The superscript $k$ will be often omitted. Note that from (2.9) it follows that

$$
e^{-M} d(x)<d(y)<e^{M} d(x)
$$

for all $y \in D(x, M)$. Moreover, one obtains from (2.9) or (2.10)

$$
\left\{\begin{array}{l}
B^{n}\left(x,\left(1-e^{-M}\right) d(x)\right) \subset D(x, M), \\
D(x, M) \subset B^{n}\left(x,\left(e^{M}-1\right) d(x)\right) .
\end{array}\right.
$$

From (2.15) and (2.16) it follows that $k_{G}$ defines the euclidean topology in $G$.

Let $f: G \rightarrow B^{n}$ be $K$-qr, $F \subset G$ compact, $\varepsilon \in(0,1)$, and let $|f(x)|<\varepsilon$ for $x \in F$. In the sequel it is important to find an appropriate upper bound for $|f(y)|$ when $y \in G$. The upper bound will be established in terms of $K, n, \delta=\operatorname{cap}(G, F)$, and $k(y, F)=\inf \{t>0: F \subset \bar{D}(y, t)\}$. The first step towards such an upper bound is the next lemma.

2.17. LemMA. Let $\lambda \in(0,1)$ and let $F \subset G$ be compact.

(1) Then there is a number $N$ depending only on $n, \lambda$, and $d(F) / d(F, \partial G)$ and points $x_{1}, \ldots, x_{p}, p \leqslant N$, in $F$ such that $F \subset \cup \cup_{j=1}^{p} B^{n}\left(x_{j}, \lambda d\left(x_{j}\right)\right)$.

(2) Suppose that $\delta=\operatorname{cap}(G, F)>0$. Then there is a point $z \in F$ such that $\operatorname{cap}\left(G, F \cap \bar{B}^{n}(z, \lambda d(z))\right) \geqslant \delta / N$.

Proof. For the proof of (1) let $p$ be the smallest number of balls $\left\{B^{n}(x, \lambda d(x))\right.$ : $x \in F\}$ needed to cover $F$. Clearly $p \leqslant q, q$ being the smallest number of balls $\left\{B^{n}(x, \lambda d(F, \partial G)): x \in F\right\}$ needed to cover $F$. From [3, p. 197, Lemma 3.2] it follows that there is a number $c(n)$, depending only on $n$, and a covering $\left\{B^{n}\left(x_{i}, \lambda d(F, \partial G)\right): i=1, \ldots, r, x_{i} \in F\right\}$ of $F$ such that at most $c(n)$ elements have common points. Obviously $r \geqslant q$. Let $F_{\lambda}=\{x \in G: d(F, x)<\lambda d(F, \partial G)\}$. We estimate

$$
r \Omega_{n}(d(F, \partial G) \lambda)^{n} \leqslant c(n) m\left(F_{\lambda}\right) \leqslant c(n) \Omega_{n}(d(F)+2 \lambda d(F, \partial G))^{n}
$$

where $\Omega_{n}=m\left(B^{n}\right)$ and where the obvious estimate $m(A) \leqslant \Omega_{n} d(A)^{n}$, valid for any measurable $A$, has been used in the last step. Thus

$$
p \leqslant r \leqslant c(n)(2+d(F) / d(F, \partial G) \lambda)^{n}=N .
$$

For the proof of (2) choose a covering $\left\{B^{n}\left(x_{i}, \lambda d\left(x_{i}\right)\right): i=1, \ldots, p\right\}$ of $F$. Then by 
(2.6) and [14, 6.2(3)]

$$
\delta=\operatorname{cap}(G, F) \leqslant \sum_{i=1}^{p} \operatorname{cap}\left(G, F \cap \bar{B}^{n}\left(x_{i}, \lambda d\left(x_{i}\right)\right)\right),
$$

and one can therefore choose $z=x_{i}$ for some $i$.

The next result is a corollary to the first part of Lemma 2.17.

2.18. Corollary. Let $F$ be a compact connected subset of $G$. Then there exists a constant $A$ depending only on $n$ and $d(F) / d(F, \partial G)$ such that $k_{G}(F) \leqslant A$.

Proof. Fix $a, b \in F$ with $k_{G}(F)=k_{G}(a, b)$. Putting $\lambda=1 / 2$ in Lemma 2.17(1) we see that there is a number $N=N(n, d(F) / d(F, \partial G))$ depending only on $n$ and $d(F) / d(F, \partial G)$ and a covering $\left\{B^{n}\left(x_{j}, d\left(x_{j}\right) / 2\right): j=1, \ldots, p\right\}, p \leqslant N$, of $F$. Since $F$ is connected, there is a subfamily of $\left\{B^{n}\left(x_{j}, d\left(x_{j}\right)\right): j=1, \ldots, p\right\}$ which is $(a, b, 1 / 2)$-admissible and contains $q$ elements. Therefore by Lemma 2.17(1)

$$
k_{G}(F)=k_{G}(a, b) \leqslant 2 q \log 2 \leqslant N \log 4 .
$$

2.19. Remarks. (1) Let $X^{+}=\left\{x \in R^{2}: x_{2}=0, x_{1} \geqslant 0\right\}$ and write $G=$ $R^{2} \backslash X^{+}$. For $t \in(0,1)$ let $a_{t}=(1, t), b_{t}=(1,-t)$, and let $F_{t}=\left\{a_{t}, b_{t}\right\}$. Then $d\left(F_{t}\right) / d\left(F_{t}, \partial G\right)=2$ for all $t \in(0,1)$ but $k_{G}\left(a_{t}, b_{t}\right) \geqslant 2 \log (1+1 / t) \rightarrow \infty$ as $t \rightarrow 0$ (cf. (2.10)). In this case $k_{G}\left(F_{t}\right)$ cannot be estimated from above in terms of $d\left(F_{t}\right) / d\left(F_{t}, \partial G\right)$ and the dimension $n=2$. Thus the assumption that $F$ be connected in Corollary 2.18 cannot be dropped.

(2) We now introduce a condition on $G$ under which one can estimate $k_{G}(F)$ from above in terms of $d(F) / d(F, \partial G)$. Suppose that there is a constant $C \geqslant 1$ such that for all $a, b \in G$ there exists a curve $\gamma \subset G$ with $a, b \in \gamma$ and $s(\gamma)<$ $C|a-b|, d(\gamma, \partial G) \geqslant C^{-1} \min \{d(a), d(b)\}$. Then it follows that $k_{G}(x, y)<$ $C^{2}|x-y| / \min \{d(x), d(y)\}$ for all $x, y \in G$ and hence $k_{G}(F) \leqslant C^{2} d(F) / d(F, \partial G)$ for any compact $F \subset G$.

(3) It is easy to show that all convex domains $G \neq R^{n}$ satisfy the condition in (2) with the constant $C=1$.

(4) In Corollary 2.18 an upper bound was obtained for $k_{G}(F)$ under the assumption that $F$ be connected. Note that a lower bound of the same type holds without any assumptions on $F$. In fact, it follows from (2.10) that

$$
k_{G}(F) \geqslant \log (1+d(F) / 2 d(F, \partial G))
$$

for all $F \subset G$.

The next result, a local version of a two-constants theorem for qr mappings, was proved by Rickman [12, 4.22] in a somewhat more general formulation. Employing some iteration ideas of Rickman [12, 4.24] and the preceding results about the quasihyperbolic geometry we shall show in Lemma 2.22 how one passes from local to global estimates.

2.20. Lemma. Let $f: G \rightarrow R^{n}$ be a nonconstant $K$-qr mapping, $0<m<M$, $U=B^{n}(z, \rho)$ and suppose

(1) $|f(x)| \leqslant M$ for $x \in G \cap U$,

(2) $\lim \sup _{x \rightarrow y}|f(x)| \leqslant m$ for $y \in \partial G \cap \bar{U}$. 
Then there are constants $\lambda_{K} \in(0,1 / 2)$ and $C>0$ depending only on $n$ and $K$ such that

$$
\log |f(x)| \leqslant \beta \log m+(1-\beta) \log M
$$

for $x \in G \cap B^{n}\left(z, \lambda_{K} \rho\right) \backslash f^{-1}(0)$ and here

$$
\beta=C\left(\operatorname{cap}\left(U, \bar{B}^{n}\left(z, \lambda_{K} \rho\right) \cap\left(R^{n} \backslash G\right)\right)\right)^{1 /(n-1)} \in[0,1] .
$$

2.21. Corollary. Let $f: B^{n}(\rho) \rightarrow B^{n}$ be $K-q r, \varepsilon \in(0,1), F \subset B^{n}(\rho)$ compact, and let $|f(x)| \leqslant \varepsilon$ for $x \in F$. If $\operatorname{cap}\left(B^{n}(\rho), \bar{B}^{n}\left(\lambda_{K} \rho / 2\right) \cap F\right) \geqslant \delta>0$ then $|f(x)|<\varepsilon^{\beta}$, $\beta=C \delta^{1 /(n-1)}$, for $x \in \bar{B}^{n}\left(\lambda_{K} \rho\right)$.

Proof. For $t \in(1 / 2,1)$ let $G_{t}$ be that component of $B^{n}(t \rho) \backslash\left(\bar{B}^{n}\left(t \lambda_{K} \rho\right) \cap F\right)$ whose boundary contains $\partial B^{n}(t \rho)$ and let $U_{t}=B^{n}(t \rho)$. Then

$$
\operatorname{cap}\left(B^{n}(t \rho), \bar{B}^{n}\left(t \lambda_{K} \rho\right) \cap\left(R^{n} \backslash G_{t}\right)\right) \geqslant \delta .
$$

Note that $f^{-1} \bar{B}^{n}\left(\varepsilon^{\beta}\right)$ is closed in $B^{n}(\rho)$. The desired upper bound follows from this observation if we apply Lemma 2.20 with $M=1, m=\varepsilon, G=G_{t}, U=U_{t}$ and let $t \rightarrow 1$.

2.22. Lemma. Let $f: G \rightarrow B^{n}$ be $K$-qr, $F \subset G$ compact, $\operatorname{cap}(G, F)>\delta>0$, and $|f(x)| \leqslant \varepsilon$ for $x \in F$. Then there is a number $\beta \in(0,1)$ depending only on $n, K, \delta$, and $d(F) / d(F, \partial G)$ such that

$$
|f(y)| \leqslant \varepsilon^{\beta^{k(y, F) c+1}},
$$

for $y \in G$ and here $k(y, F)=\inf \{t>0: F \subset \bar{D}(y, t)\}, c=\left(\log \left(1+\lambda_{K}\right)\right)^{-1}$.

Proof. Fix $y \in G$. Let $\lambda=\lambda_{K} \in(0,1 / 2)$ be as in Lemma 2.20 and let $N=$ $N(n, \lambda / 2, d(F) / d(F, \partial G))$ be the number and $z \in F$ the point given by Lemma 2.17. Choose a $(z, y, \lambda)$-admissible chain of balls as in the chaining lemma, Lemma 2.14 , with the centers $x_{1}=z, x_{2}, \ldots, x_{p}, x_{j+1} \in \partial B^{n}\left(x_{j}, \lambda d\left(x_{j}\right)\right)$, and with $y \in$ $\bar{B}^{n}\left(x_{p}, \lambda d\left(x_{p}\right)\right)$,

$$
p \leqslant 1+k_{G}(z, y)(\log (1+\lambda))^{-1} \leqslant 1+k(y, F)(\log (1+\lambda))^{-1} .
$$

We now stepwise use Corollary 2.21 and obtain the following estimates.

STEP 1. Let $F_{1}=\bar{B}^{n}\left(x_{1}, \lambda d\left(x_{1}\right) / 2\right) \cap F$. By the choice of $z=x_{1}$ $\operatorname{cap}\left(B^{n}\left(x_{1}, d\left(x_{1}\right)\right), F_{1}\right) \geqslant \delta / N$. Corollary 2.21 gives now $|f(x)|<\varepsilon^{\beta_{0}}, \quad \beta_{0}=$ $C(\delta / N)^{1 /(n-1)}$ for $x \in \bar{B}^{n}\left(x_{1}, \lambda d\left(x_{1}\right)\right)$.

STEP 2. Let $F_{2}=\bar{B}^{n}\left(x_{2}, \lambda d\left(x_{2}\right) / 2\right) \cap \bar{B}^{n}\left(x_{1}, \lambda d\left(x_{1}\right)\right)$. By the chaining lemma it follows that $(1+\lambda) d\left(x_{j}\right) \geqslant d\left(x_{j+1}\right) \geqslant(1-\lambda) d\left(x_{j}\right)$ and therefore $F_{2} \cap S^{n-1}\left(x_{2}, r\right)$ $\neq \varnothing$ for $r \in\left(0, \lambda d\left(x_{2}\right) / 2\right)$. Now one obtains by [17, 1.10] or [14, 10.9]

$$
\operatorname{cap}\left(B^{n}\left(x_{2}, d\left(x_{2}\right)\right), F_{2}\right) \geqslant c_{n} \log (2 /(2-\lambda)) \geqslant c_{n}(\log (1+\lambda)) / 2 .
$$

Let $\beta_{1}=C\left(\left(c_{n} / 2\right) \log (1+\lambda)\right)^{1 /(n-1)}$ and $\beta=\min \left\{\beta_{0}, \beta_{1}\right\}$. Corollary 2.21 yields

$$
|f(x)| \leqslant\left(\varepsilon^{\beta_{0}}\right)^{\beta_{1}} \leqslant \varepsilon^{\beta^{2}} \text { for } x \in \bar{B}^{n}\left(x_{2}, \lambda d\left(x_{2}\right)\right) .
$$

STEP $j$. Proceeding as in Step 2 we obtain

$$
|f(x)| \leqslant\left(\varepsilon^{\beta^{j-1}}\right)^{\beta_{1}} \leqslant \varepsilon^{\beta^{j}}, \quad j=2, \ldots, p,
$$


for $x \in \bar{B}^{n}\left(x_{j}, \lambda d\left(x_{j}\right)\right)$, which yields the desired estimate.

In the following result we shall show that the dependence on $F$ of the constant $\beta$ in Lemma 2.22 can be expressed in terms of $k_{G}(F)$.

2.23. Corollary. Let $f: G \rightarrow B^{n}$ be a $K$-qr mapping, let $\varepsilon \in(0,1)$, let $F \subset G$ be compact, and let $|f(x)| \leqslant \varepsilon$ for $x \in F$. Write $\delta=\operatorname{cap}(G, F)>0$ and $M_{0}=k_{G}(F)$ and let $M>0$. Then there is a constant $\gamma \in(0,1)$ depending only on $n, K, M_{0}$, and $\delta$ such that

$$
|f(x)| \leqslant \varepsilon^{\gamma^{\left(M_{0}+M\right) c+1}}
$$

for $x \in \cup_{z \in F} D(z, M)$ and here $c=\left(\log \left(1+\lambda_{K}\right)\right)^{-1}$.

Proof. By inspecting the proof of Lemma 2.22 we see that it will be enough to obtain an upper bound for the number $N=N\left(n, \lambda_{K}, d(F) / d(F, \partial G)\right)$ in Lemma 2.17(1) in terms of $n, \lambda_{K}$, and $k_{G}(F)$. By the proof of Lemma 2.17(1) $N$ is increasing as a function of $d(F) / d(F, \partial G)$ and thus it is enough to have an upper bound for $d(F) / d(F, \partial G)$. The estimate (cf. Remark 2.19(4))

$$
d(F) / d(F, \partial G) \leqslant 2\left(e^{k_{G}(F)}-1\right)
$$

yields the desired upper bound. The proof now follows from the proof of Lemma 2.22 , since $k(y, F) \leqslant M_{0}+M$ for all $y \in \cup_{z \in F} D(z, M)$.

3. Bounded quasiregular mappings. In the present section we shall apply the results in $\$ 2$ to prove a theorem concerning angular limits of bounded qr mappings. The boundedness condition will then be weakened in $\$ 4$.

For $E \subset R^{n}, x \in R^{n}$, and $r>0$ we write

$$
M(E, r, x)=M\left(\Delta\left(S^{n-1}(x, 2 r), \bar{B}^{n}(x, r) \cap E ; R^{n}\right)\right)
$$

and define the lower and upper capacity densities of $E$ at $x$ by

$$
\begin{aligned}
& \text { cap } \underline{\operatorname{dens}}(E, x)=\liminf _{r \rightarrow 0} M(E, r, x), \\
& \text { cap } \overline{\operatorname{dens}}(E, x)=\underset{r \rightarrow 0}{\lim \sup } M(E, r, x) .
\end{aligned}
$$

For examples illustrating the condition cap $\operatorname{dens}(E, x)>0$ the reader is referred to [5], [9], and [17].

In this section and the following one we shall use the quasihyperbolic metric of $B^{n}$, which we shall denote by $k$. It is easily seen that the ordinary hyperbolic metric $\rho$ of $B^{n}$ and $k$ are equivalent. In fact, $\rho \leqslant k \leqslant 2 \rho$, if $\rho$ has the element of length $d \rho=|d x|\left(1-|x|^{2}\right)^{-1}$.

For $b \in S^{n-1}$ and $\varphi \in(0, \pi / 2)$ let $K(b, \varphi)$ be the open bounded cone $\{x$ : $(b \mid b-x)>|b-x| \cos \varphi\} \cap B^{n}(b, \cos \varphi)$. Here $(x \mid y)$ is the inner product $\sum_{i=1}^{n} x_{i} y_{i}$.

3.1. THEOREM. Let $f: B^{n} \rightarrow B^{n}$ be a qr mapping, $b \in S^{n-1}, \varphi_{0} \in(0, \pi / 2)$, and let $E \subset K\left(b, \varphi_{0}\right)$. If $f(x)$ tends to 0 when $x$ approaches $b$ through $E$ and if cap $\operatorname{dens}(E, b)=\delta>0$ then $f$ has angular limit 0 at $b$, i.e. $\lim _{x \rightarrow b, x \in K(b, \varphi)} f(x)=0$ for all $\varphi \in(0, \pi / 2)$. 
Proof. Fix $\varphi \in\left(\varphi_{0}, \pi / 2\right)$. Let $\lambda=\lambda(n, \delta) \in(0,1)$ be such that $\omega_{n-1}(\log \lambda)^{1-n}$ $<\delta / 3$. Here $\omega_{n-1}$ is the $(n-1)$-dimensional surface area of $S^{n-1}$. Fix $\varepsilon>0$. Choose $r_{\varepsilon} \in(0, \cos \varphi)$ with $f\left(B^{n}\left(b, r_{\varepsilon}\right) \cap E\right) \subset B^{n}(\varepsilon)$ and $M(E, r, b) \geqslant 2 \delta / 3$ for $r \in\left(0, r_{\varepsilon}\right)$. Let $r_{1}=r_{\varepsilon}, r_{j+1}=\lambda r_{j}, R_{j}=K(b, \varphi) \cap R\left(b, r_{j}, \lambda r_{j}\right), j=1,2, \ldots$ Using a simple continuity argument we may assume that $E_{j}=E \cap \bar{R}_{j}$ is compact (cf. [17, 3.2]). Let $F_{1}=E_{j}, F_{2}=\partial B^{n} \cap B^{n}\left(b, r_{j}\right)$, and $F_{3}=S^{n-1}\left(b, 2 r_{j}\right)$. Employing the notation of (2.4) we get by the choice of $\lambda$ (cf. [14, 7.5]) $M\left(\Gamma_{13}\right) \geqslant \delta / 3$, and by [14, 10.12] $M\left(\Gamma_{23}\right) \geqslant c_{n} \log 2$. Hence we obtain by (2.4)

$$
\operatorname{cap}\left(B^{n}, E_{j}\right) \geqslant M\left(\Gamma_{12}\right) \geqslant c(n, \delta)>0
$$

where $c(n, \delta)$ depends only on $n$ and $\delta$. By elementary geometry (cf. [17, 6.12])

$$
d\left(R_{j}, \partial B^{n}\right) \geqslant 1-\sqrt{\sin ^{2} \varphi+\left(\cos \varphi-\lambda r_{j}\right)^{2}} \geqslant\left(\lambda r_{j} \cos \varphi\right) / 3 .
$$

Since $d\left(R_{j}\right) \leqslant 2 r_{j}$ it follows from Remark 2.19(3) that

$$
k\left(R_{j}\right) \leqslant d\left(R_{j}\right) / d\left(R_{j}, \partial B^{n}\right) \leqslant 6 /(\lambda \cos \varphi) .
$$

Lemma 2.22 now gives

$$
|f(x)| \leqslant \varepsilon \quad \text { for } x \in \bar{R}_{j},
$$

where $T>0$ depends only on $K, n, \delta$, and $\varphi$. Therefore

$$
|f(x)| \leqslant \varepsilon^{T} \quad \text { for } x \in \bigcup_{j=1}^{\infty} \bar{R}_{j} \backslash\{0\}
$$

and the proof is complete, since $\varepsilon>0$ is arbitrary.

3.2. REMARKs. (1) By Rickman's example $[13,1.1]$ the nontangentiality condition $E \subset K(b, \varphi)$ cannot be dropped for dimensions $n \geqslant 3$. On the other hand it follows from [13,1.3] that this condition can be dropped for $n>2$ if $E$ is a piece of an $(n-1)$-dimensional surface having a nice behavior at $b$. Note that there are sets $E$ with cap dens $(E, b)>0$ which have Hausdorff dimension zero [17, 2.5(3)].

(2) The condition cap $\operatorname{dens}(E, b)>0$ in Theorem 3.1 cannot be replaced by cap $\overline{\operatorname{dens}}(E, b)>0$ (cf. [17, 6.6]).

(3) It is possible that Theorem 3.1 is new even for analytic functions in the complex plane because of the generality of the assumption cap dens $(E, b)>0$. It is not known to the author whether the nontangentiality condition $E \subset K(b, \varphi)$ can be dropped in this particular case. In the case of bounded analytic functions, $T$. Hall has proved a result of this kind without a nontangentiality condition but assuming a condition stronger than cap $\operatorname{dens}(E, b)>0$. References to Hall's work and to related results of J. L. Doob can be found in [17, §\$4 and 5] (cf. also in [11, p. 65]).

By Rickman's result [13, Theorem 1.1] a qr mapping of $B^{n}, n>3$, into itself need not have an angular limit at a boundary point even if it has an asymptotic value $\alpha$ at the point in question. However, the values of the mapping converge towards $\alpha$ through a rather large set as the following theorem shows.

3.3. Theorem. Let $f: B^{n} \rightarrow B^{n}$ be qr, $b \in \partial B^{n}$, and let $E \subset B^{n}$ be a connected set with $b \in \bar{E}$ and $\lim _{x \rightarrow b, x \in E} f(x)=0$. Fix $t>0$ and let $E_{t}=\left\{x \in B^{n}: k(E, x)<\right.$ $t$. Then $\lim _{x \rightarrow b, x \in E_{t}} f(x)=0$. 
Proof. The proof follows directly from Corollary 2.23 or Lemma 2.22.

The condition that the set $E$ be connected in Theorem 3.3 cannot be dropped, as one can easily show by considering a modified version of the exponential function. In fact, the failure of the statement of Theorem 3.3 for a disconnected set $E$ with $b \in \bar{E}$ follows from the example given in $[17,6.4]$, where $E$ consisted of a sequence of $a$-points of the function, converging towards a boundary point (cf. also [18, 4.5]).

4. Nonbounded quasiregular mappings. In this section we shall weaken the boundedness assumption in Theorem 3.1 by requiring only that the set of omitted values $\bar{R}^{n} \backslash f B^{n}$ be thick enough at $\infty$. The continuum criterion of Martio [4] provides us with an appropriate thickness condition. The basic idea here is to reduce the proof to the case of bounded mappings by use of the capacity inequality (2.7) and then apply Theorem 3.1.

Let $D$ be a domain in $\bar{R}^{n}=R^{n} \cup\{\infty\}$ with $\infty \in \partial D$. Let $t>0$ with $D \cap$ $S^{n-1}(t) \neq \varnothing$. For $r>t$ let

$$
M_{r}=\inf M(\Delta(C, \partial D ; D))
$$

where the infimum is taken over all continua $C \subset D$ with $C \cap S^{n-1}(t) \neq \varnothing$ and $C \cap S^{n-1}(r) \neq \varnothing$. Then we write $M\left(\infty, \bar{R}^{n} \backslash D\right)=\infty$ if $\lim _{\inf _{r \rightarrow \infty}} M_{r}=\infty$ (cf. Martio [4]). Otherwise $M\left(\infty, \bar{R}^{n} \backslash D\right)<\infty$. It is not difficult to show that the condition is independent of a number $t$ with $D \cap S^{n-1}(t) \neq \varnothing$ (cf. [4], [9]). At a point $x \in \partial D \backslash\{\infty\}$ the conditions $M\left(x, \bar{R}^{n} \backslash D\right)=\infty$ and $M\left(x, \bar{R}^{n} \backslash D\right)<\infty$ are defined in an analogous way [4], [9].

4.1. THEOREM. Let $f: B^{n} \rightarrow R^{n}$ be a qr mapping, $b \in S^{n-1}, \varphi_{0} \in(0, \pi / 2)$, and let $E \subset K\left(b, \varphi_{0}\right)$. Suppose that cap dens $(E, b)=\delta>0$ and $\lim _{x \rightarrow b, x \in E} f(x)=\alpha$. If

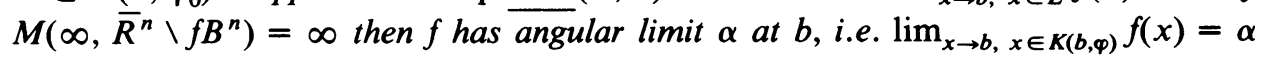
for all $\varphi \in(0, \pi / 2)$.

Proof. Fix $\varphi \in\left(\varphi_{0}, \pi / 2\right)$. As in the proof of Theorem 3.1 choose $\lambda \in(0,1)$ such that $\omega_{n-1}(\log \lambda)^{1-n}<\delta / 3$. Fix $r_{1} \in(0, \cos \varphi)$ such that $M(E, r, b) \geqslant 2 \delta / 3$ for all $r \in\left(0, r_{1}\right]$. Let $r_{j+1}=\lambda r_{j}$ and $R_{j}=K(b, \varphi) \cap R\left(b, r_{j}, \lambda r_{j}\right), j=1,2, \ldots$ From the choice of $\lambda$ it follows that $M\left(E \cap R_{j}, r_{j}, b\right) \geqslant \delta / 3$ for all $j$ (cf. the proof of Theorem 3.1). For each $j=1,2, \ldots$, fix $x_{j} \in E \cap R_{j}$.

CASE 1. $\alpha=\infty$. Suppose that the theorem is not true. Then we find a sequence $\left(y_{j}\right)$ in $K(b, \varphi)$ with $\left|f\left(y_{j}\right)\right|<t<\infty$ for all $j$. By passing to a subsequence and by relabeling if necessary we may assume that $y_{j} \in R_{j}$ and $\left|f\left(x_{j}\right)\right|>\max \{j, t\}$ for all $j$. It was shown in the proof of Theorem 3.1 that

$$
d\left(R_{j}\right) / d\left(R_{j}, \partial B^{n}\right) \leqslant 6 /(\lambda \cos \varphi) .
$$

From this estimate and $[14,8.2]$ it follows that

$$
M\left(\Delta\left(\bar{R}_{j}, \partial B^{n} ; B^{n}\right)\right) \leqslant a
$$

where $a \in(0, \infty)$ does not depend on $j$. By (2.6) and (2.7) we obtain

$$
\operatorname{cap}\left(f B^{n}, f \bar{R}_{j}\right) \leqslant K(f) \operatorname{cap}\left(B^{n}, \bar{R}_{j}\right) \leqslant K(f) a
$$


which yields a contradiction, since $M\left(\infty, \bar{R}^{n} \backslash f B^{n}\right)=\infty$ and $S^{n-1}(t) \cap f \bar{R}_{j} \neq \varnothing$ $\neq S^{n-1}(j) \cap f \bar{R}_{j}$ for all large $j$.

CASE 2. $|\alpha|<\infty$. Fix $\theta \in(\varphi, \pi / 2)$. We shall first show that $|f(x)|$ is bounded when $x \in K(b, \theta) \cap U$ where $U$ is a neighborhood of $b$. Suppose that this is not the case. Then we find a sequence $\left(y_{j}\right)$ in $K(b, \theta)$ with $y_{j} \rightarrow b$ and $f\left(y_{j}\right) \rightarrow \infty$ as $j \rightarrow \infty$. By passing to a subsequence and by relabeling if necessary we may assume that $\left|f\left(x_{j}\right)\right|<|\alpha|+1$ and $y_{j} \in R_{j}^{\theta}, R_{j}^{\theta}=K(b, \theta) \cap R\left(b, r_{j}, \lambda r_{j}\right)$ for all $j$. Making use of the condition $M\left(\infty, \bar{R}^{n} \backslash f B^{n}\right)=\infty$ in the same way as in the proof of Case 1 we get a contradiction. Hence there exists a neighborhood $U$ of 0 such that $|f(x)|$ is bounded when $x \in K(b, \theta) \cap U$. The proof of the theorem can now be carried out in the same way as in the case of Theorem 3.1.

4.2. Remarks. (1) Some examples illustrating the condition $M\left(x, \bar{R}^{n} \backslash D\right)=\infty$ can be found in [4] and [9].

(2) If $G$ is a domain with $B^{n} \subset G$ and if $b \in \partial G \cap \partial B^{n}$ and if $R^{n} \backslash G$ is so thin at $b$ that $M\left(b, R^{n} \backslash G\right)<\infty$, then a qr mapping $f: G \rightarrow R^{n}$ with $\operatorname{cap}\left(\bar{R}^{n} \backslash f G\right)>0$ always has an angular limit at 0 (cf. [16, §8]).

(3) Martio and Rickman [6, 5.17] have proved a result related to Theorem 4.1 by a different method.

(4) Results stronger than Theorem 4.1 are known for qc mappings [17, §§4 and 5] and for qr local homeomorphisms ([18], for dimensions $n \geqslant 3$ ).

\section{REFERENCES}

1. F. W. Gehring and B. G. Osgood, Uniform domains and the quasi-hyperbolic metric, J. Analyse Math. 36 (1979), 50-74.

2. F. W. Gehring and B. P. Palka, Quasiconformally homogeneous domains, J. Analyse Math. 30 (1976), 172-199.

3. N. S. Landkof, Foundations of modern potential theory, Die Grundlehren der math. Wissenschaften, Band 180, Springer-Verlag, Berlin, New York and Heidelberg, 1972.

4. O. Martio, Equicontinuity theorem with an application to variational integrals, Duke Math. J. 42 (1975), 569-581.

5. 109-118.

6. O. Martio and S. Rickman, Boundary behavior of quasiregular mappings, Ann. Acad. Sci. Fenn. Ser. A I Math. 507 (1972), 1-17.

7. O. Martio, S. Rickman and J. Väisälä, Definitions for quasiregular mappings, Ann. Acad. Sci. Fenn. Ser. A I Math. 448 (1969), 1-40.

8. Distortion and singularities of quasiregular mappings, Ann. Acad. Sci. Fenn. Ser. A I Math. 465 (1970), 1-13.

9. O. Martio and J. Sarvas, Density conditions in the n-capacity, Indiana Univ. Math. J. 26 (1977), 761-776.

10. R. Näkki, Extension of Loewner's capacity theorem, Trans. Amer. Math. Soc. 180 (1973), 229-236.

11. M. Ohtsuka, Dirichlet problem, extremal length, and prime ends, Van Nostrand Reinhold, New York, 1970.

12. S. Rickman, On the number of omitted values of entire quasiregular mappings, J. Analyse Math. 37 (1980), 100-117.

13. Asymptotic values and angular limits of quasiregular mappings of a ball, Ann. Acad. Sci. Fenn. Ser. A I Math. 5 (1980), 185-196.

14. J. Väisälä, Lectures on n-dimensional quasiconformal mappings, Lecture Notes in Math., vol. 229, Springer-Verlag, Berlin, New York and Heidelberg, 1971.

15. _ A survey of quasiregular maps in $R^{n}$, Proc. 1978 Internat. Congr. Math. (Helsinki, Finland), Finnish Academy of Science and Letters, Helsinki, 1980. 
16. M. Vuorinen, Exceptional sets and boundary behavior of quasiregular mappings in n-space, Ann. Acad. Sci. Fenn. Ser. A I Math. Dissertationes 11 (1976), 1-44.

17. On the existence of angular limits of n-dimensional quasiconformal mappings, Ark. Mat. (to appear)

18. , On the boundary behavior of locally $K$-quasiconformal mappings in space, Ann. Acad. Sci. Fenn. Ser. A I Math. 5 (1980), 79-95.

19. L__ Lindelof-type theorems for quasiconformal and quasiregular mappings, Proc. Complex Analysis Semester, Banach Center, Warsaw, Poland, 1979 (to appear).

20. W. P. Ziemer, Extremal length and p-capacity, Michigan Math. J. 16 (1969), 43-51.

Department of Mathematics, University of Michigan, AnN Arbor, Michigan 48109

Current address: Department of Mathematics, University of Helsinki, Hallituskatu 15, 00100 Helsinki 10, Finland 